Abstracted/indexed in Academic Search Complete, Asia Journals Online, Bangladesh Journals Online, Biological Abstracts, BIOSIS Previews, CAB

Abstracts, Current Abstracts, Directory of Open Access Journals, EMBASE/Excerpta Medica, Google Scholar, HINARI (WHO), International

\title{
Anticancer activity of midostaurin in hormone refractory human prostate cancer DU145 cells
}

\author{
Jin-Jun Sun, Shi-Feng Kan and Guan-Xing Sun
}

Department of Oncology, Zaozhuang Municipal Hospital, Shandong 277 101, P. R. China.

\begin{tabular}{|c|c|}
\hline \multicolumn{2}{|l|}{ Article Info } \\
\hline Received: & 10 September 2015 \\
\hline Accepted: & 22 October 2015 \\
\hline Available Online: & 24 March 2016 \\
\hline \multicolumn{2}{|c|}{ DOI: 10.3329/bjp.v11i2.24954 } \\
\hline \multicolumn{2}{|c|}{$\begin{array}{l}\text { Cite this article: } \\
\text { Sun JJ, Kan SF, Sun GX. Anticancer } \\
\text { activity of midostaurin in hormone } \\
\text { refractory human prostate cancer } \\
\text { DU145 cells. Bangladesh J Pharmacol. } \\
\text { 2016; } 11 \text { : 378-82. }\end{array}$} \\
\hline
\end{tabular}

\begin{abstract}
We tried a new method of prostate cancer treatment by inducing in vitro differentiation which resulted in reduction of cancer cells growth. A protein kinase inhibitor, midostaurin's ability to trigger the human prostate cancer cell line, DU145 to segregate into nerve cells was studied. Midostaurin (100 $\mathrm{nM}$ ) suppressed the growth of DU145 cells but without change in the number of dead cells. Midostaurin started to extend neurites on DU145 cells after 24 hours and differentiated into nerve cells by 72 hours. The microtubule was stabilized by tau protein and its mRNA expression showed time-dependent increase in midostaurin-treated DU145 cells. At the same time, the amount of acetylcholinesterase was also increased. The midostaurin-treated DU145 cells showed $40 \%$ less activity than control in the colony forming assay. The results suggests that midostaurin can induce differentiation of DU145 cells into nerve cells.
\end{abstract}

\section{Introduction}

Prostate cancer in men is the major type of cancers which counts for $27 \%$ of all the cancer cases diagnosed. Androgen receptor signaling is an important step in progression of cancer cells and for differentiation of normal prostate cells (Gelmann, 2002). Even though there are benefits in androgen ablation therapy in prostate carcinoma, it becomes hormone refractory in the advanced stages of prostate cancer (Lepor et al., 1982).

Taxanes are used as the most effect chemotherapeutic agents used in hormone refractory prostate cancer treatment, but many patients develop acquired resistance after treatment. An alternate way of treatment by differentiating the cells were studied with leukemia cells (Koeffler, 1983; Hozumi, 1998). Reagents such as butyrate analogues and cytokines have shown to reduce the tumor in prostate cancer cell lines (Bang et al., 1994; Gleave et al., 1998; Mori et al., 1999; Nishimura et al., 1998) but with less success in inducing cell differentiation (Bang et al., 1994; Itayasu et al., 1998).

Midostaurin is a multi-target protein kinase inhibitor and an alkaloid obtained from the bacterium Streptomyces staurosporeus. Studies of midostaurin showed it reduces multiplication of tumor cells during the starting phase of cell cycle by triggering apoptosis, polyploidy and sensitive to radio waves and inhibition of PI3K/ Akt pathway inhibition (Tenzer et al., 2001). In this study, we examined the ability of midostaurin in the treatment of human prostate cancer DU145 cells.

\section{Materials and Methods}

\section{Chemicals and reagents}

RPMI 1640 medium and MTT were purchased from Life Technologies (USA). PVDF membrane and chemiluminescent reagents were from EMD Millipore (USA). Fetal bovine serum and midostaurin was obtained from Sigma-Aldrich (USA) and dimethyl sulfoxide is used to prepare a $10 \mathrm{mM}$ stock and stored at $-20^{\circ} \mathrm{C}$. All other reagents and chemicals used in this study were of analytical grade.

\section{Cell culture and proliferation assay}


The human prostate carcinoma cell line DU145 was purchased from American Type Culture Collection (USA). Cells were grown in RPMI 1640 medium with $10 \%$ heat-inactivated fetal bovine serum as supplementary. The cells were incubated at $37^{\circ} \mathrm{C}$ in incubator with $5 \% \mathrm{CO}_{2}$. The concentration-based effect of midostaurin for the proliferation of DU145 cells was performed by pre-culturing the cells for one day at $37^{\circ} \mathrm{C}$ and midostaurin ( $1 \mathrm{nM}$ to $1 \mathrm{mM}$ ) was added and incubated for 72 hours. $1 \% \mathrm{NaCl}$ was used to wash DU145 cells (Sunrise, USA). The proliferation of midostaurin treated DU145 cells was measured at various time periods, by seeding 10,000 cells per dish and incubated 24 hours at $37^{\circ} \mathrm{C}$. After 24 hours, $100 \mathrm{nM}$ of midostaurin was added to the cells. The number of viable cells was identified by using tryphan blue methods with the help of hemocytometer for up to 7 days.

\section{Phenotypic measurements}

Midostaurin (100 nM) was added to DU145 cells for one week at a density of 10,000 cells per dish. The morphological changes were observed by measuring the length and diameter of the cell. After the cells were treated with midostaurin, about 500 cells were measured for its size under the phase-contrast microscope (Carl-Zeiss, Switzerland).

\section{Soft-agar colony formation}

DU145 cells in 6-well plates (1,000 cells/well) at single cell density were used for colony forming assay. The cells were treated with $100 \mathrm{nM}$ of midostaurin for one week and then collected. The washed cells were again seeded in $0.4 \%$ soft agar. $r$-iodonitro tetrazolium violet solution was used to stain the cells after two weeks. Colonies consisting of more than 50 cells considered for counting.

\section{Cell invasion assay}

Midostaurin (100 nM) was added to DU145 cells for 7 days were harvested and used for invasion assay. The cell invasion assay was carried out by using MaxGel ECM (Sigma-Aldrich) by measuring the invasion of the treated cells compared to control group. The basement membrane extract diluted in cold DMEM medium and incubated for $3-4$ hours at $37^{\circ} \mathrm{C}$ in a $\mathrm{CO}_{2}$ incubator. The remaining solution was aspirated before the cells were plated. It was air dried for $30 \mathrm{~min}$ at room temperature and the cells were plated. The cell penetration was measured by staining the cells with Giemsa stain and using microscope the penetrated cells were counted.

\section{Protein extraction}

The total proteins from DU145 cells were isolated by using RIPA buffer containing $1 \%$ nonidet-P40 substitute, $150 \mathrm{mM}$ sodium chloride, $0.1 \%$ SDS, $0.5 \%$ sodiumdeoxycholate, $50 \mathrm{mM}$ Tris pH 8.0 and $1 \mathrm{mM}$ EDTA. 10\% PMSF and $1 \%$ protease inhibitor was added as supplement. The cells were harvested and homogenized with
RIPA buffer and incubated for $30 \mathrm{~min}$ in ice. After incubation, the cells were centrifuged at 10,000 xg for 2 min. Total proteins were separated and quantification of proteins was done by using Bradford assay reagent (Bio-Rad)

\section{Western blot}

The midostaurin treated cells were lysed in lysis buffer by repeated freeze thaw cycles and by melting. The concentration of the total protein was determined with Bio-Rad protein assay (Bio-Rad Laboratories, USA). The cell extracts were denatured and analyzed by SDSPAGE. The protein extracts were separated by $12.5 \%$ SDS-PAGE and transferred to PVDF membrane (Life Technologies, USA). 5\% non-fat milk was used to block the blots overnight in TBS. Next day, the membrane was incubated with anti-acetylcholinesterase antibody at room temperature. The membrane with proteins were then washed thrice with TBS and 0.05\% Tween-20. After washing, it was incubated with HRP-conjugated secondary antibody raised in goat. The proteins which are immunoreactive were detected by ECL reagent (Image Quant LAS4000, GE Healthcare Systems, USA).

\section{Preparation of total RNA and real-time PCR}

The total cellular RNA from midostaurin treated DU145 cells was prepared by using TRI reagent (SigmaAldrich, USA) following the instruction from the manufacturer. The total RNA was assessed quantitatively in a spectrophotometer at 260 and $280 \mathrm{~nm}$ (NanoVue Plus, GE Healthcare Life Sciences, USA) and qualitatively by agarose gel electrophoresis. The RNA was reverse transcripted into cDNA using Moloney murine leukemia virus reverse transcriptase kit (Life Technologies, USA) as per the instructions from the manufacturer. In order to attain good results, tau primer was used in PCR with the sense: CATGCCAGACCTGAAGAATG and antisense: GAGTCTACCATGTCGATGCT. The polymerase chain reaction was performed for 30 cycles at $94^{\circ} \mathrm{C}$ for $30 \mathrm{sec}$, $55^{\circ} \mathrm{C}$ for $1 \mathrm{~min}$, and $72^{\circ} \mathrm{C}$ for $2 \mathrm{~min}$. To normalize gene expression, few internal controls were used such as the b-2 microglobulin, GADPH or $18 \mathrm{sec}$ were used. qPCR was performed in Mini Option real-time PCR machine (Bio-Rad laboratories, USA). Several dilutions of cDNA of DU145 cells were used to determining amplification efficiency for all primer sets using. Melting curve analysis was used to determine the concentration of the primers and the optimum temperature for annealing for all primer sets and the ampion specificity. The PCR products were confirmed by DNA sequencing and also by $1.0 \%$ agarose gel at $100 \mathrm{~V}$ for $30 \mathrm{~min}$ and stained with SYBR green (Life Technologies, USA).

\section{Results}

Midostaurin suppresses proliferation of DU145 cells 
Midostaurin inhibited proliferation of DU145 cells in dose-dependent manner in the concentrations up to 100 $\mathrm{nM}$ (Figure 1A). At $100 \mathrm{nM}$ concentration, midostaurin totally inhibited the growth of DU145 cells in one week (Figure 1B). The number of dead cells on day 7 was almost the same as that of control. Midostaurin also dose-dependently suppressed two other hormone refractory prostate cancer cell lines, TSU-Pr1 and PPC-1. But there was no morphological changes induced by the treatment of midostaurin.

\section{Midostaurin induces neurite outgrowth in DU145 cells}

The phase contrast microscopic studies of $100 \mathrm{nM}$ midostaurin treated DU145 cells showed that the morphological changes were seen in neurons (Figure 2A). Neurite extension started to appear within 24 hours and after 72 hours, most of the cells turned into cells with neuronal characteristics (Figure 2B).

\section{Expression of neuronal markers in midostaurin-treated} cells

To observe DU145 cells have any neuronal characteristics after treating with $100 \mathrm{nM}$ of midostaurin, the expression of typical neuronal markers such as tau and acetylcholinesterase (AchE) were studied. Time-dependent increase in tau mRNA expression was observed in $100 \mathrm{nM}$ midostaurin treated cells (Figure 3A) and concomitant increase in AchE protein was also observed (Figure 3B).

\section{Midostaurin treatment reduces malignancy}

The effect of midostaurin treatment on malignancy of DU145 cells was investigated by performing colony forming assay in soft agarose gel and invasion assay in in vitro. The DU145 cells treated with $100 \mathrm{nM}$ of midostaurin shows $40 \%$ less colony forming activity than that of control (Figure 4A). $100 \mathrm{nM}$ of midostaurin

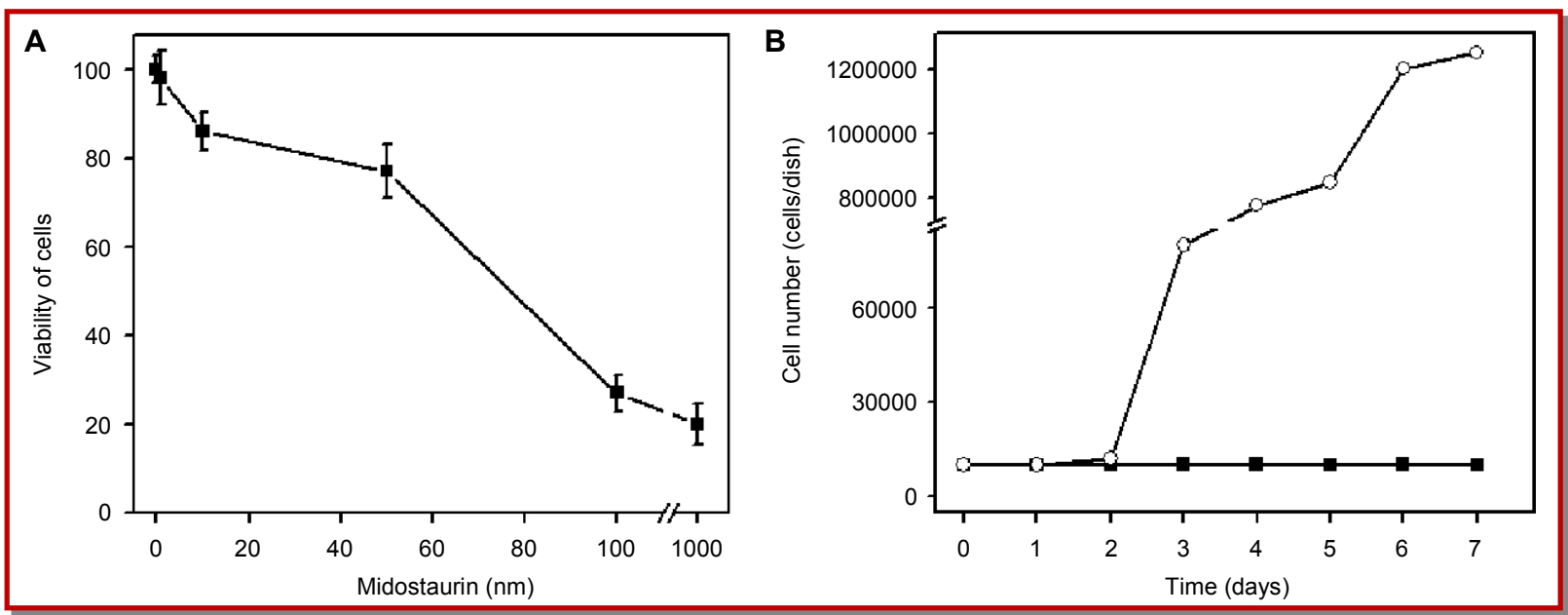

Figure 1: A. Concentration-dependent effect of midostaurin on DU145 cells proliferation. Cells were treated with midostaurin for 72 hours. Viable cells are calculated as per the standard protocol. T. B. Midostaurin effect on proliferation of DU145 cells. The values given are mean \pm SD of three independent experiments

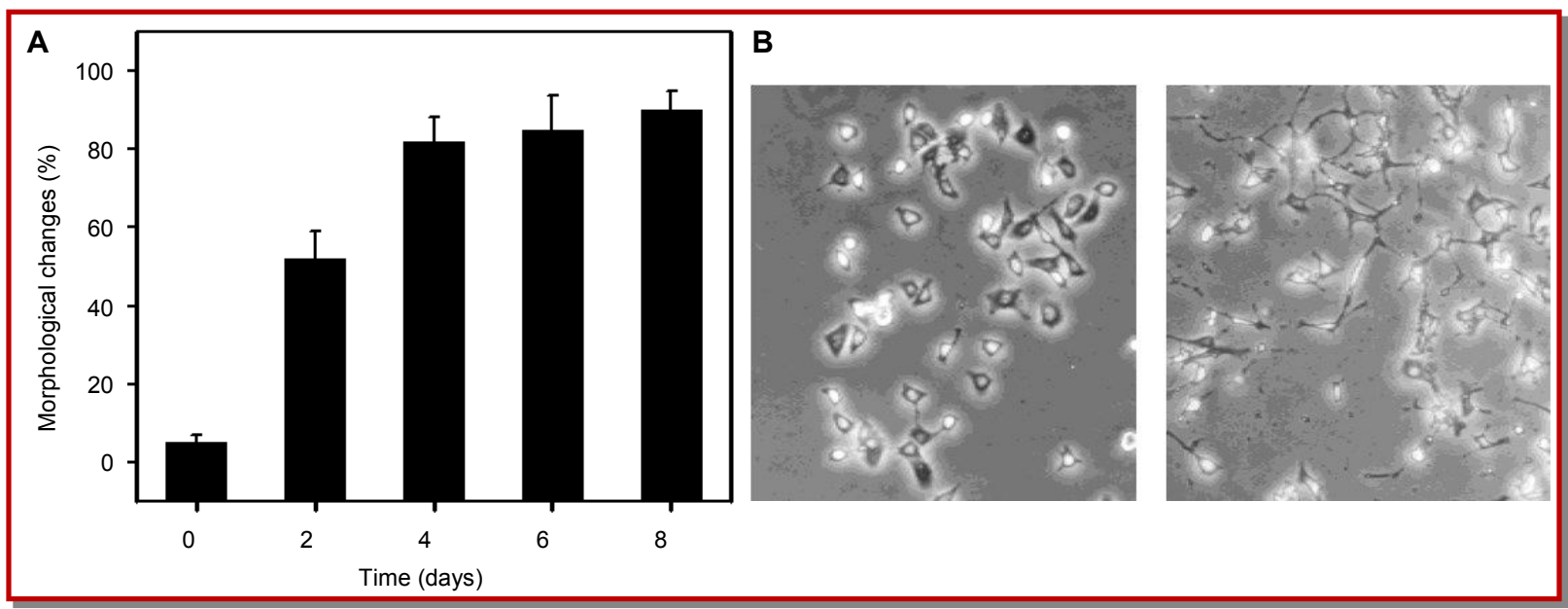

Figure 2: A. Neuronal characteristics of DU145 cells with $100 \mathrm{nM}$ midostaurin treatment at different time intervals. The values given are the mean of triplicate experiments with standard deviation. B. Microscopic observations of the morphological appearances in DU145 cells. Phase-contract microscopic images of DU145 cells (i) untreated and $100 \mathrm{nM}$ midostaurin treated cells for (ii) for 72 hours 
treated DU145 cells showed 20\% invasive ability than the untreated cells (Figure 4B).

\section{Discussion}

We investigated the anticancer properties of midostaurin in DU145 cells. The midostaurin treatment in the human prostate cancer cell lines, DU145 cells showed decreased in cell proliferation and also induced neurite outgrowth. Previously studies in another prostate cancer cell line, TSU-Pr1 showed that cell proliferation was suppressed by 12-O-tetradecanoylphorbol-13-acetate changes in the phenotype such as amoeboid shape and a-naphthyl acetate esterase activity (Itayasu et al., 1998). As TPA is a known PKC activator, effects of various PKC inhibitors were studied to know whether PKC plays an essential role in signal transduction which leads to differentiation. GF109203X is a specific inhibitor of PKC was able to block the TPA induced differentiation (data not shown). Midostaurin, a protein kinase inhibitor has not blocked the segregation of DU145 cells inducing TPA but was able to suppress the

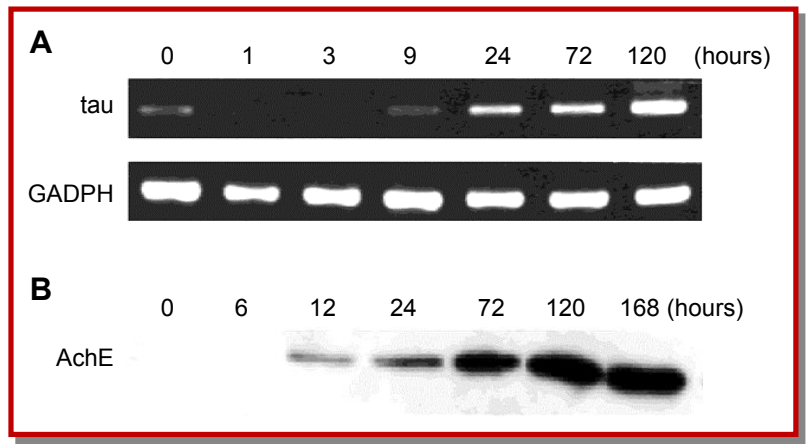

Figure 3: A. The midostaurin treatment effect on the expression of tau mRNA in DU145 cells at various time intervals. B. Timedependent effect of midostaurin on acetylcholinesterase protein content in DU145 cells

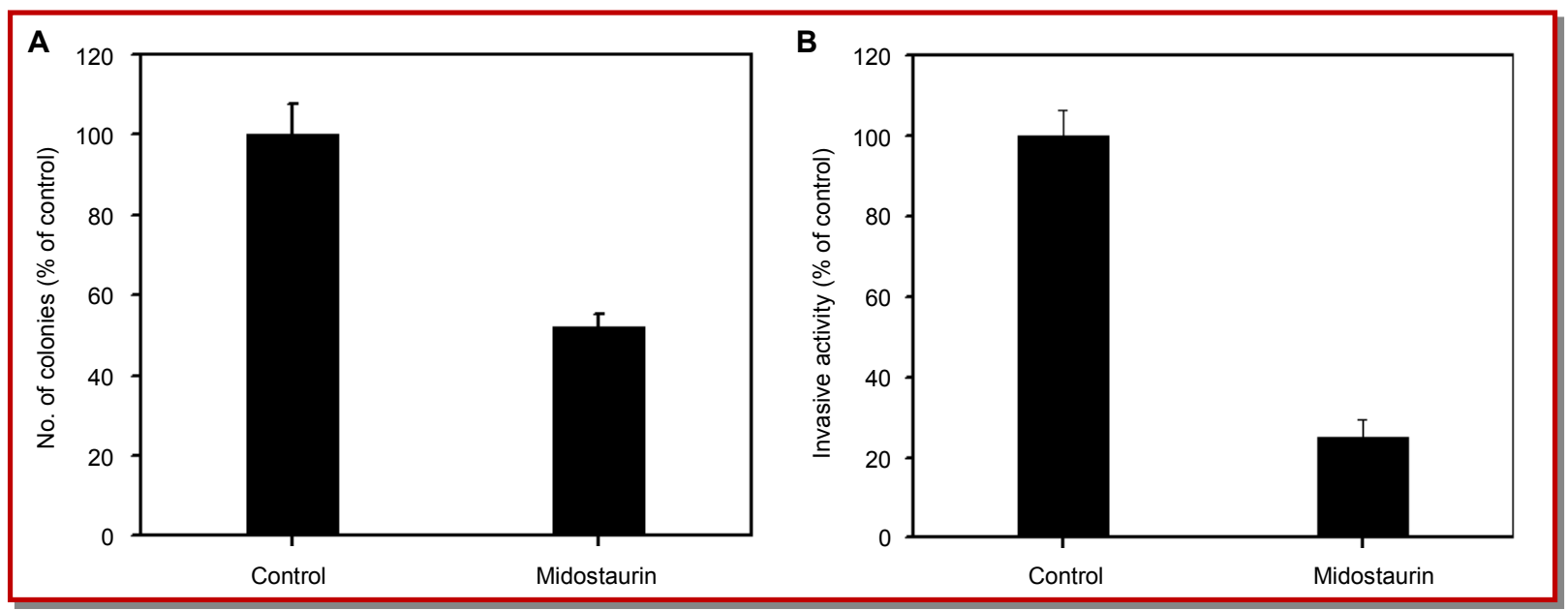

Figure 4: A. Colony forming activity assay on midostaurin treated DU145 cells for one week. Values are given as mean \pm SD of three independent experiments. B. Effects of midostaurin on invasive activity of DU145 cells. Values are mean \pm SD of triplicate experiments growth of DU145 cells at $10 \mathrm{mM}$ concentration but inhibited the action of TPA (data not shown). The above result suggests that proliferation of DU145 cells was suppressed by midostaurin and the suppression was not due to the PKC inhibition.

We found that midostaurin treatment resulted in neurite outgrowth in DU145 cells. It was responsible for inducing expression of mRNA content of tau protein. tau is capable of making an axonal shape and an axonlike organization of the cytoskeleton (Kosik, 1993). Another report suggested that AchE may play a role in differentiation of neuronal cells (Koenigsberger et al., 1997). Protein kinase inhibitors are reported to be involved in inducing neuronal outgrowth in neuronal cell lines (Sasaki et al., 1997; Rasouly et al., 1993; Rasouly et al., 1992). Since midostaurin is also a protein kinase inhibitor, the possibilities are high that midostaurin may induce differentiation in DU145 cells into cells with neuronal characteristics. Other protein kinase inhibitors are reported to induce human prostate cancer cell lines to differentiate into neuronal cells and results in apoptosis (Zhang et al., 1996). However this study did not detect the changes in morphology in protein kinase inhibitor treated DU145 cells. These results might be because of the various concentration of the inhibitors used in the study. Even though, the action of midostaurin treatment on DU145 cells are mainly by the protein kinase inhibition, but their underlying pathway remains to be explored. TPA treated cells induced differentiated the cells with the microglial characteristics but the midostaurin treated cells induced differentiation of cells to neuronal characteristics in the

\section{Conclusion}

Midostaurin was able to suppress the proliferation of human prostate cancer cell line, DU145. 
DU145 cells completely for 1 week without any change in viability. The midostaurin-treated cells shows decrease in colony formation and invasive activity in in vitro studies. These findings explain that midostaurin may help to reduce the malignancy of human prostate cancer DU145 cells. This study revealed that inducing the prostate cancer cells differentiating into neuronal cells will be an efficient way of treatment for hormone refractory prostate cancer.

\section{References}

Bang YJ, Pirnia F, Fang WG, Kang WK, Sartor O, Whitesell L, Ha MJ, Tsokos M, Sheahan MD, Nguyen P, Niklinki WT, Myers CE, Trepel JB. Terminal neuroendocrine differentiation of human prostate carcinoma cells in response to increased intracellular cyclic AMP. Proc Natl Acad Sci USA. 1994; 91: 5330-34.

Gelmann EP. Molecular biology of the androgen receptor. J Clin Oncol. 2002; 20: 3001-15.

Gleave ME, Sato N, SadarM, Yago V, Bruchovsky N, Sullivan L. Butyrate analogue, isobutyramide, inhibits tumor growth and time to androgen-independent progression in the human prostate LNCaP tumor model. J Cell Biochem. 1998; 69: 271-81.

Hozumi M. Differentiation therapy of leukemia: Achievements, limitations and future prospects. Int J Hematol. 1998; 68: $107-29$

Itayasu $\mathrm{T}$, Shimizu T, Iizumi T, Oshio S, Umeda T, Takeda $\mathrm{K}$. Phorbol ester induces differentiation of a human prostatic cell line TSU-Pr1 into cells with characteristics of microglia. Anticancer Res. 1998; 18: 113-17.

Koeffler HP. Induction of differentiation of human acute myelogenous leukemia cells: Herapeutic implications. Blood 1983; 62: 709-21.

Koenigsberger C, Chiappa S, Brimijoin S. Neurite differentiation is modulated in neuroblastoma cells engineered for altered acetylcholinesterase expression. J Neurochem. 1997; 69: 1389-97.
Kosik KS. The molecular and cellular biology of tau. Brain Pathol. 1993; 3: 39-43.

Lepor H, Ross A, Walsh PC. The influence of hormonal therapy on survival of men with advanced prostatic cancer. J Urol. 1982; 128: 335-40.

Mori S, Murakami-Mori K, Bonavida B. Interleukin-6 induces G1 arrest through induction of p27/kip1, a cyclin-dependent kinase inhibitor, and neuron-like morphology in LNCaP prostate tumor cells. Biochem Biophys Res Commun. 1999; 257: 609-14.

Nishimura K, Kitamura K, Takada S, Nonomura N, Tsujimura A, Matsuyama K, Miki T, Matsumoto K, Okuyama A. Regulation of invasive potential of human prostate cancer cell lines by hepatocyte growth factor. Int J Urol. 1998; 5: 276-81.

Rasouly D, Rahamim E, Lester DS, Matsuda Y, Lazalovici P. Staurosporine-induced neurite outgrowth in PC12 cells is independent of protein kinase $\mathrm{C}$ inhibition. Mol Pharmacol. 1992; 42: 35-43.

Rasouly D, Rahamim E, Ringel I, Ginzburg I, Murakata C, Matsuda Y, Lazarovici P. Neurites induced by staurosporine in PC12 cells are resistant to colchicine and express high levels of tau proteins. Mol Pharmacol. 1993; 45: 29-35.

Sasaki K, Maruyama K, Nishimura E, Tsukada T, Yamaguchi K. Differentiation of cultured neuroblastoma induced by staurosporine and cyclic AMP: Methods for assessing a neuronal phenotype. Brain Res Protoc. 1997; 1: 399-405.

Tenzer A, Zingg D, Rocha S, Hemmings B, Fabbro D, Glanzmann C, Shubiger PA, Bodis S, Pruschy M. The phosphatidylinositide 30-kinase/Akt survival pathway is a target for the anticancer and radiosensitizing agent PKC412, an inhibitor of protein kinase C. Cancer Res. 2001; 1: 3-10

Zhang H, Hoang T, Saeed B, Ng SC. Induction of apoptosis in prostatic tumor cell line DU145 by staurosporine, a potent inhibitor of protein kinases. Prostate 1996; 29: 69-76. 


\section{Your feedback about this paper}

1. Number of times you have read this paper 0

2. Quality of paper

$\bigcirc$ Excellent $\quad \bigcirc$ Good $\quad \bigcirc$ Moderate $\quad \bigcirc$ Not good

3. Your comments

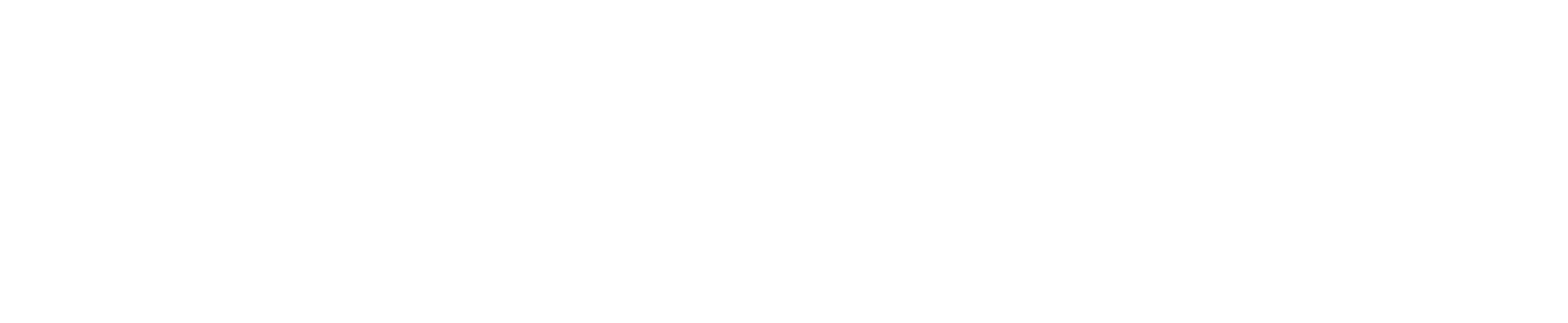

